\title{
Search for global oscillations on Jupiter with a double-cell sodium magneto-optical filter
}

\author{
A. Cacciani ${ }^{1}$, M. Dolci ${ }^{1,2}$, P. F. Moretti ${ }^{1,3}$, F. D'Alessio ${ }^{4}$, C. Giuliani ${ }^{2}$, E. Micolucci $^{2}$, and A. Di Cianno $^{2}$ \\ 1 Università degli Studi di Roma "La Sapienza", P.le A. Moro 5, 00185 Rome, Italy \\ 2 Osservatorio Astronomico "V. Cerulli" di Teramo, Via M. Maggini, 64100 Teramo, Italy \\ 3 Osservatorio Astronomico di Capodimonte, Via Moiariello 16, 80131 Napoli, Italy \\ 4 Osservatorio Astronomico di Roma, Via Frascati 33, 00040, Monte Porzio Catone, Italy
}

Received 30 January 2001 / Accepted 22 March 2001

\begin{abstract}
Doppler observations on Jupiter are presented and discussed. A two-cells Magneto-Optical Filter (MOF), able to obtain two separate signals, Red and Blue, on the opposite wings of the Sodium D-lines, along with a continuum reference signal, has been used. This set of data permits to discriminate between real oscillations and albedo "modes" by means of the two algorithms $\mathcal{D}=(B-R) /(B+R)$ and $\mathcal{S}=(B+R) /$ continuum. No unambiguous oscillation modes were detected with amplitudes above the $1-\sigma$ level of $\sim 1.2 \mathrm{~m} \mathrm{~s}^{-1}$ in the range between 0.5 and $0.7 \mathrm{mHz}$. However, using refined analysis for signal recovery in a noisy background we notice an increase of power also in the region of the solar $5 \mathrm{~min}$ oscillations. The albedo variations on the Jupiter's surface and instrumental effects are addressed to be responsible for the spurious signals.
\end{abstract}

Key words. planets and satellites: general

\section{Introduction}

During the last decade an increasing interest has been devoted to Jovian seismology, as a powerful tool to investigate the interior of the planet (Vorontsov et al. 1976; Bercovici \& Schubert 1987). Also, thanks to its brightness, it is an intermediate case between the Sun and the stars. Besides the theoretical modelling (Lee 1992, 1993; Mosser 1990, 1994, 1995), only few observations have been carried out so far (Deming et al. 1989; Schmider et al. 1991; Mosser et al. 1993, 2000).

Observed at about $10 \mu \mathrm{m}$ along the equator, Jupiter showed no oscillations with an amplitude above $1 \mathrm{~m} \mathrm{~s}^{-1}$ (Deming et al. 1989). Nevertheless, a non-acoustic thermal wave structure already present in the Voyager data (Magalhães et al. 1991; Pirraglia et al. 1992) has been reported by several authors (Harrington et al. 1996a, 1996b; Magalhães et al. 1989). Recent observations with infrared cameras have confirmed these preliminary conclusions (Deming et al. 1997).

Observations in the Sodium D-lines were performed using a simple system based on the Magneto-Optical Filter (MOF) by Schmider et al. (1991). The MOF is a filter that provides two very narrow and close passbands

Send offprint requests to: M. Dolci, e-mail: dolci@astrte.te.astro.it (about $50 \mathrm{~m} \AA$ each) called $B$ (lue) and $R($ ed) because, in the solar case, they are located on the opposite wings of the Fraunhofer line. For the Jovian case, Schmider et al. (1991) did not operate any selection between $B$ and $R$ and since the overall filter passband $B+R$ is narrower than the typical line width, any displacement or deformation in its profile is received as intensity variations. The signal was normalized to a wide band continuum in order to compensate for atmospheric transmission fluctuations. Five consecutive nights of integrated disk observations showed a power spectrum with peak amplitudes ranging from 4 to $8 \mathrm{~m} \mathrm{~s}^{-1}$, later identified as possible $\ell=1$ and $\ell=2$ $p$-modes (Mosser et al. 1991).

The same result was obtained by observing a limited portion of the Jupiter's surface through a Fourier transform spectrometer. Mosser et al. (1993) have used a disk of just 12 arcsec in diameter. Their data consist of two sets of four consecutive nights covering about 500 lines in the whole methane- $3 \nu_{3}$ band around $1.1 \mu \mathrm{m}$. Essentially, the same structure was found in the power spectrum, with similar amplitudes.

In principle these results are not contradictory. As pointed out by Lederer, those different observational techniques are sensitive to different oscillation modes (Lederer et al. 1995; Dolci \& Cacciani 2001, in preparation); moreover they sample different heights in the Jovian 
atmosphere $(\sim 0.5$ bar by Deming et al. $1989, \sim 1.3$ bar by Mosser et al. 1993 and $\sim 3$ bar by Schmider et al. 1991); therefore some physical process could be invoked for the modes that are visible at the level investigated by Schmider and Mosser but damped at the level of Deming (whose observations are not really velocities, but refer to infrared intensities, i.e. to the temperature). Alternatively, other phenomena could be considered to reproduce the power spectra so far obtained, and indeed Lederer et al. (1995) proved that quasi-periodical albedo variations could severely contaminate the power spectrum.

In this paper we present in detail our original observational technique and careful analysis of a sample of calibrated data obtained during a 3 day observation campaign. The adopted technique is illustrated in Sect. 2. It is currently used in Helioseismology and was succesfully tested on Jupiter during the impact of the Comet $\mathrm{P} /$ Shoemaker-Levy 9 (Cacciani et al. 1995). The instrument used by Schmider et al. (1991) is a simplified version of it. The data and the calibration procedures are presented in Sect. 3, while their analysis is discussed in Sect. 4 . In Sect. 4 we also compare our results with a recent report from Mosser et al. (2000) that sets an upper limit of $60 \mathrm{~cm} \mathrm{~s}^{-1}$ for the $p$-mode amplitudes.

\section{Observational technique}

\subsection{The filter}

The core of the experiment is the MOF, described in several papers (Cacciani \& Fofi 1978; Cacciani et al. 1988, 1991; Rhodes et al. 1990; Tomczyk et al. 1995). Here we briefly recall its working principle.

In the MOF a cloud of metallic vapors embedded in a magnetic field acts as a wavelength-dependent depolarizing medium so that transmission occurs when interposed between two crossed polarizers.

The version developed by Cacciani is composed by two fundamental units. The first one is properly called the MOF itself and transmits two narrow passbands, called $B$ and $R$, located on the opposite wings of the solar sodium$\mathrm{D}$ lines at $589 \mathrm{~nm}$. With an appropriate choice of the vapors' temperature and the magnetic field $\left(250-300{ }^{\circ} \mathrm{C}\right.$ and

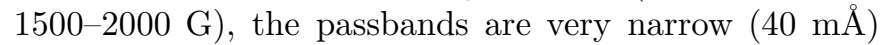
and displaced by approximately $180 \mathrm{~mA}$ : the peak transmission is typically $40 \%$ (it cannot be higher than $50 \%$, because of the first polarizer).

The second unit is called Wing Selector $(W S)$ because permits the separation of the two bands by forcing their polarization to become opposite. Thereafter, a selection can be operated sequentially, as in this work and in the MOF installed at Mount Wilson (Rhodes et al. 1990), or simultaneously (as suggested for the MOF used at HAO (Tomczyk et al. 1995)). Just like the MOF itself, the WS consists of a cloud of metallic vapors at about $300{ }^{\circ} \mathrm{C}$, but embedded in a fairly stronger magnetic field (2500-3000 G).
The use of the sole first unit allows single band photometry $(B+R$ normalized to the continuum $)$ as in Schmider et al. (1991). When the second cell is also used, two separate wavelengths are available and differential measurements can be performed. This is the Doppler technique widely used in Helioseismology $(B-R$ normalized to $B+R$; we also use $\log R-\log B$ ) and adopted for the first time on Jupiter during the impacts of Comet SL9 (Cacciani et al. 1995). Its applicability to the stellar case, however, is seriously constrained by the very limited photon flux and velocity biases. Stellar observations with magneto-optical techniques have been attempted in the past (Gelly et al. 1986; Innis et al. 1991, 1994), but the analysis is rather difficult, except in the case of strong pulsators (Brown \& Gilliland 1994).

\subsection{Experimental set-up}

The experimental set-up that we have used in 1996 (Moretti et al. 1999) is illustrated in Fig. 1. It is essentially the same as used to observe the SL9-Jupiter impacts (Cacciani et al. 1995) with some important improvements. The light coming from the telescope is divided in two beams by a polarization beam-splitter: the first one passes through the $\mathrm{MOF}+\mathrm{WS}$ and the transmitted light is collected by a photon counting device, while the second one, which would be rejected in any case by the first MOF polarizer, is used to feed the CCD tracking camera and a photodiode for the continuum reference channel.

The main improvement consists on the presence of this reference signal. Therefore we have three separate channels, $B, R$ and continuum, from which we derive both the differential quantity $\mathcal{D}=(B-R) /(B+R)$ and the "monochromatic" intensity $\mathcal{S}=(B+R) /$ continuum. The three algorithms $B /$ cont., $R /$ cont. and $B / R$ can also be considered. Other important improvements include high transmission anti-reflecting coated optics, fast electrooptical modulators and a temperature control within $1{ }^{\circ} \mathrm{C}$.

The overall system is computer controlled to synchronize the optical modulator with the acquisition sequence and to record the MOF's and WS's temperatures. The timing is provided by a GPS antenna.

The optical alignment can be checked visually first, then looking for the maximum counts of the detector.

\subsection{Functionality tests}

A few simple tests were performed at the start of each observing run.

The first one was, of course, to verify the signal transmission when the MOF is turned on: a count increase should be observed. Then the WS was also turned on, and the expected signal decrease of about $40 \%$ (essentially due to the suppression of most of one band) was observed. However, this general behavior could be partially contaminated by thermal photons coming from the MOF heater. This possibility can be checked (and eventually corrected 


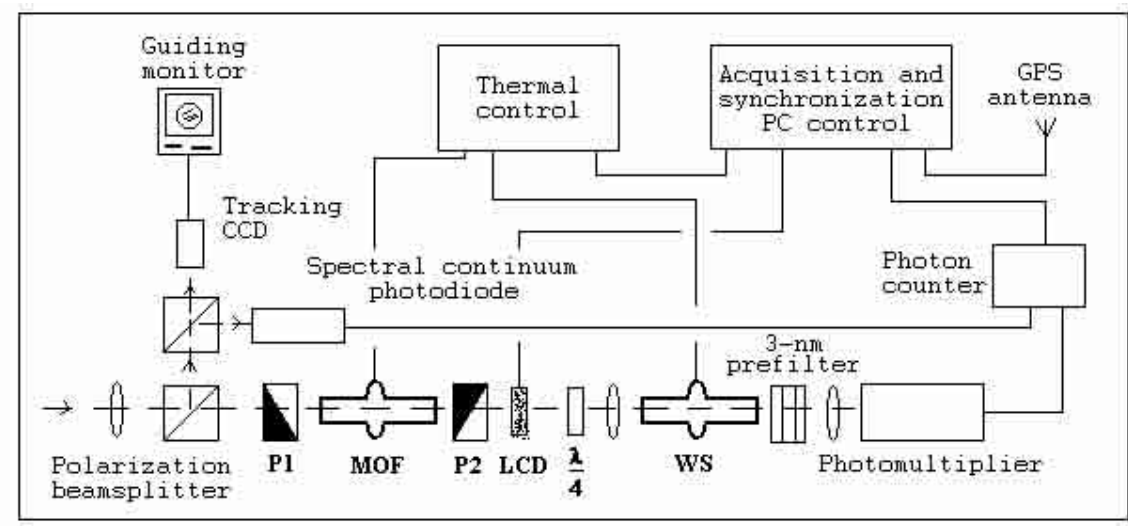

Fig. 1. The experimental set-up. Light is coming from the left. The beam is divided by a polarization beamsplitter. Half the light passes through the Magneto-Optical Filter (MOF) and the Wing Selector (WS) and is refocused on a photomultiplier. A narrow-band $(30 \AA)$ interference filter selects only the portion of the spectrum around the lines of interest. The second half is split further and feeds a tracking CCD and a secondary channel which is devoted to monitor the sky transparency fluctuations. Because of the very low light levels, photon counting technique is needed. The overall system is synchronized and controlled by a computer which is interfaced with a GPS antenna.

by suitable baffling) by observing the passage of Jupiter across the field of view, as in Fig. 4a. This test is also necessary to verify the overall optical alignment, and will be used later in the discussion about data calibration.

As a final test, we also observed the sky in a region near the Moon, in order to receive a photon flux of solar origin, large enough to be detected. The different behavior of this "sky" solar signal and the "Jovian" signal confirmed the correct tuning of the MOF by verifying the non-zero value of their differential signal $B-R$, as explained in the Sect. 2.4. Typical levels of dark current were $70-80$ counts per second.

\subsection{Acquisition procedure}

The Jovian line is usually found substantially shifted because of the relative Earth/Jupiter orbital motion: therefore a systematic difference between $B$ and $R$ is expected because they are tuned on the same flank of it. This quantity is approximately constant during each day and is different for the solar and the Jovian case. It can be used to set the integration time of a single measurement long enough (order of seconds) for unambiguous detection of the signals, but still shorter than the expected Jovian characteristic fluctuations $(\sim 1 / 1000 \mathrm{~Hz})$.

$B$ and $R$ raw data are recorded alternatively every second; then $\mathcal{D}$ (and the other combinations) are summed up over $30 \mathrm{~s}$ to increase the $\mathrm{S} / \mathrm{N}$ ratio. The reference signal is acquired continously.

\section{Data calibration}

\subsection{Theoretical calibration}

Our data were obtained at the $72-\mathrm{cm}$ Ritchey-Chrétien T.N.T. Telescope of the Osservatorio Astronomico di Collurania-Teramo (OACT) during three nights, from 11 to 13 July, 1996.
The limited photon flux makes detection of small velocity signals rather difficult in the Jovian and the stellar case. Therefore, different calibration methods have been conceived and compared.

The first method is the simplest one and consists of theoretical computations of the MOF's response to small shifts of the unperturbed spectral line. The results are shown in Fig. 2 for the signal $\mathcal{D}=(B-R) /(B+R)$.

Assuming $\mathcal{D}$ linear with the velocity $v$,

$\mathcal{D}=a_{1} v+a_{2}$

the $a_{1}, a_{2}$ coefficients are easily computed and the values $a_{1} \simeq 10^{-6} \mathrm{~m}^{-1} \mathrm{~s}, a_{2} \simeq-0.0131$ are obtained for the time of the observation $\left(v \simeq-3 \mathrm{~km} \mathrm{~s}^{-1}\right)$.

However, the true MOF profiles are not monitored during the run and therefore this computation is largely uncertain. Moreover, because of the strong rotation of the planet, this method does not represent exactly what happens to its spectrum. Indeed, a surface perturbation does not shift the Jovian line as a whole but, rather, it is responsible for a complex distortion of its profile (see Fig. 2 in Cacciani et al. 1995). This fact causes a deviation of $a_{1}$ from the value quoted above, depending on the epoch of the observation: higher values are expected during the opposition, lower values during the quadrature (see Dolci \& Cacciani 2001 for complete numerical simulations).

\subsection{Earth's rotation method}

Once the raw data have been reduced and the signals $\mathcal{D}$ and $\mathcal{S}$ computed, a general trend due to the Earth's rotation is visible in the time-series (Fig. 3) (some discrepancies, visible around $0^{\mathrm{h}}$ and $50^{\mathrm{h}}$ as an offset of $50 \mathrm{~m} \mathrm{~s}^{-1}$, are due to different adjustments in the electronics during the acquisition of the continuum).

This signature can be used to calibrate directly the time-series, once the total velocity along the line-of-sight is known. For this, we need to consider the Earth's spin 


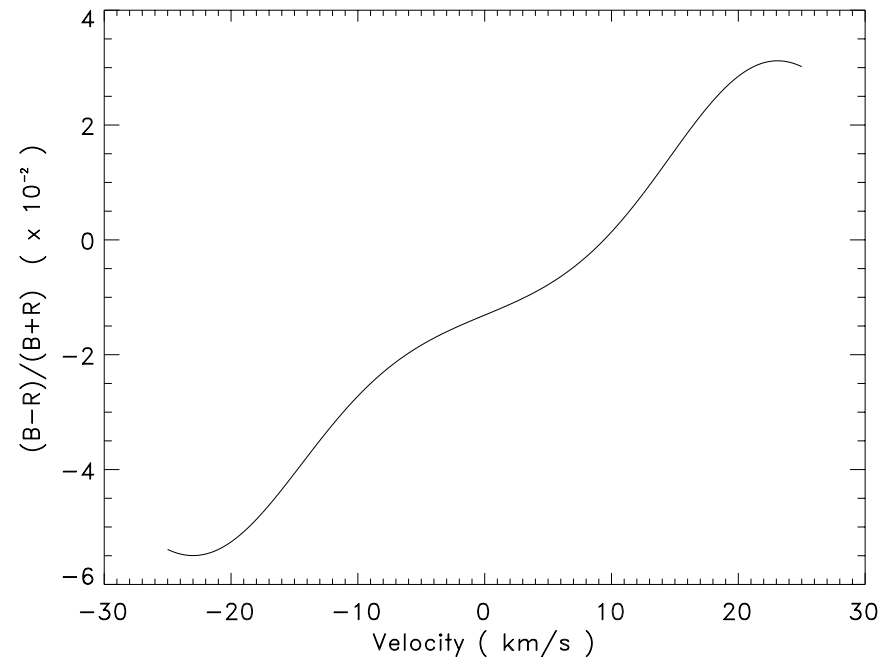

Fig. 2. The theoretical calibration curve of the signal $\mathcal{D}=$ $a_{1} v+a_{2}$. This curve is obtained considering first the unperturbed Jovian line profile, then shifting it across the MOF's $B$ and $R$ transmission profiles and finally plotting $\mathcal{D}=(B-$ $R) /(B+R)$ versus $v$. The detailed shape of the curve depends on the Jovian as well as $B$ and $R$ MOF's transmission profiles. It is not fully linear, but for calibration purposes only its central part is needed, which is fairly linear. This method provides $a_{1} \simeq 10^{-6} \mathrm{~m}^{-1} \mathrm{~s}$. However, one must consider that it is not representative of what really happens, because the $p$-mode oscillations do not shift the Jovian line as a whole. Moreover, Jupiter reflects the solar spectrum so that any Doppler shift appears doubled (not accounted for here).

velocity and the relative Earth/Jupiter orbital motion: the former is merely sinusoidal with an amplitude of about $400 \mathrm{~m} \mathrm{~s}^{-1}$, the latter is rapidly varying at the opposition, at the rate of about $2.5 \mathrm{~km} \mathrm{~s}^{-1}$ in 3 days.

This method applies to $\mathcal{D}$ as well as $\mathcal{S}=(B+R) /$ continuum, which can be also regarded as a linear function of $v$ :

$\mathcal{S}=b_{1} v+b_{2}$

The values $a_{1}=9.126 \times 10^{-6} \mathrm{~m}^{-1} \mathrm{~s}$ and $b_{1}=3.886 \times$ $10^{-4} \mathrm{~m}^{-1} \mathrm{~s}$ are obtained. The value of $a_{1}$ is one order of magnitude larger than the theoretical estimate, as expected and pointed in Sect. 3.1.

\subsection{Passage of Jupiter across the field of view}

A new method consists in recording the $B$ and $R$ signals during the passage of Jupiter across the field of view, and then comparing the associated quantities $\mathcal{D}$ and $\mathcal{S}$ with what expected theoretically.

The principle underlying this method is the following. When Jupiter crosses the field of view, only a portion of its surface is visible at a time, leading primarily to two consequences: 1) the rotation velocity, averaged over the visible portion, is a non-null signal (it is zero only when the full disk is visible); 2) the combined spectral line is strongly distorted. As a result, $\mathcal{D}$ and $\mathcal{S}$ are changing during the

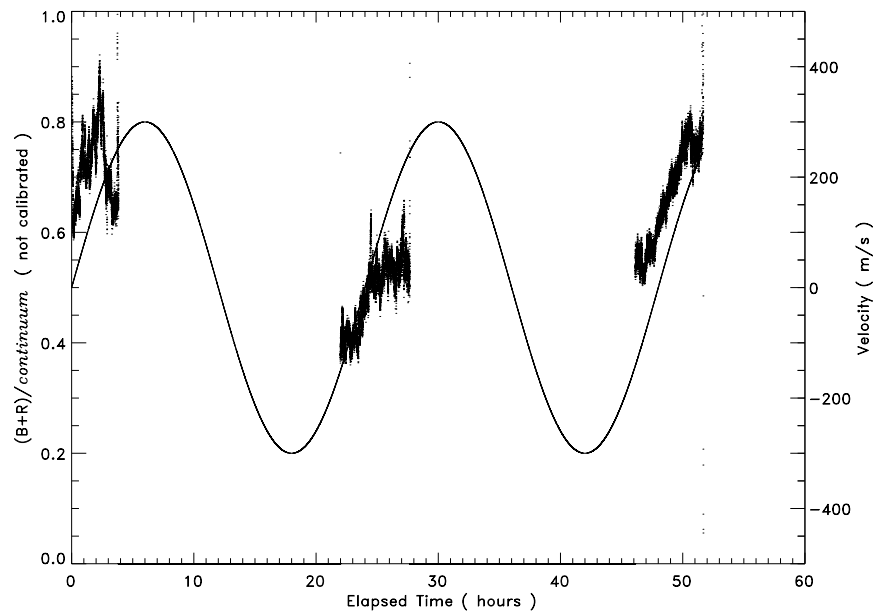

Fig. 3. The signature due to the Earth's rotation (the orbital contribution has been detrended here) is already visible in $\mathcal{S}=(B+R) /$ continuum. The offset visible around $0^{\mathrm{h}}$ and $50^{\mathrm{h}}$ is due to variations, from one night to the other, in the initial sensitivity set-up of the continuum photodiode (to prevent saturation). Once corrected for this effect, this trend has been used to calibrate the data, leading to $a_{1}=9.126 \times 10^{-6} \mathrm{~m}^{-1} \mathrm{~s}$ and $b_{1}=3.886 \times 10^{-4} \mathrm{~m}^{-1} \mathrm{~s}$. More uncertain estimates have been obtained for $a_{2}$ and $b_{2}$ : they are, however, irrelevant because represent a constant offset which does not influence the frequency pattern of the power spectrum.

passage and their values are related to the averaged velocity. In particular when just the extreme limb of Jupiter is visible, the signal is a measurement of its peripheral rotation velocity.

When using this method, attention was devoted to the presence of straylight around Jupiter which could produce a wrong result mixing together signals from the whole disk. In other words, while the direct image is vignetted, the diffused contribution is not. However, a simple estimate of this contribution shows that it is negligible. To see it, consider a fixed direction on the sky, close to Jupiter: from this direction, the terrestrial atmosphere sees different regions of Jupiter under slightly different scattering angles, within an aperture $\Delta \theta \simeq 40$ arcsec. If Rayleigh scattering is assumed, the distribution function of the scattered light is given by $P(\cos \theta)=\tilde{\omega}\left(1+a \cos ^{2} \theta\right)$, where the single-scattering albedo $\tilde{\omega}$ and the factor $a$ do not influence the normalized signals. The variation of this distribution within $\Delta \theta$ is, neglecting $\tilde{\omega}$ and $a$,

$\Delta P \sim \sin \theta \Delta \theta \sim(\Delta \theta)^{2} \simeq 3.76 \times 10^{-8}$

which corresponds to a contribution of few $\mathrm{mm} \mathrm{s}^{-1}$, given the limb-to-limb Doppler signal of $\sim 50 \mathrm{~km} \mathrm{~s}^{-1}$ (in the reflected sunlight).

The presence of optical vignetting is also very dangerous because the intensity map and the Doppler map are distorted differently. This is exactly what happened to a preliminary set of data taken at Table Mountain Observatory by N. Murphy of JPL using the setup of Fig. 1 and the hardware provided by us. For this reason, special 

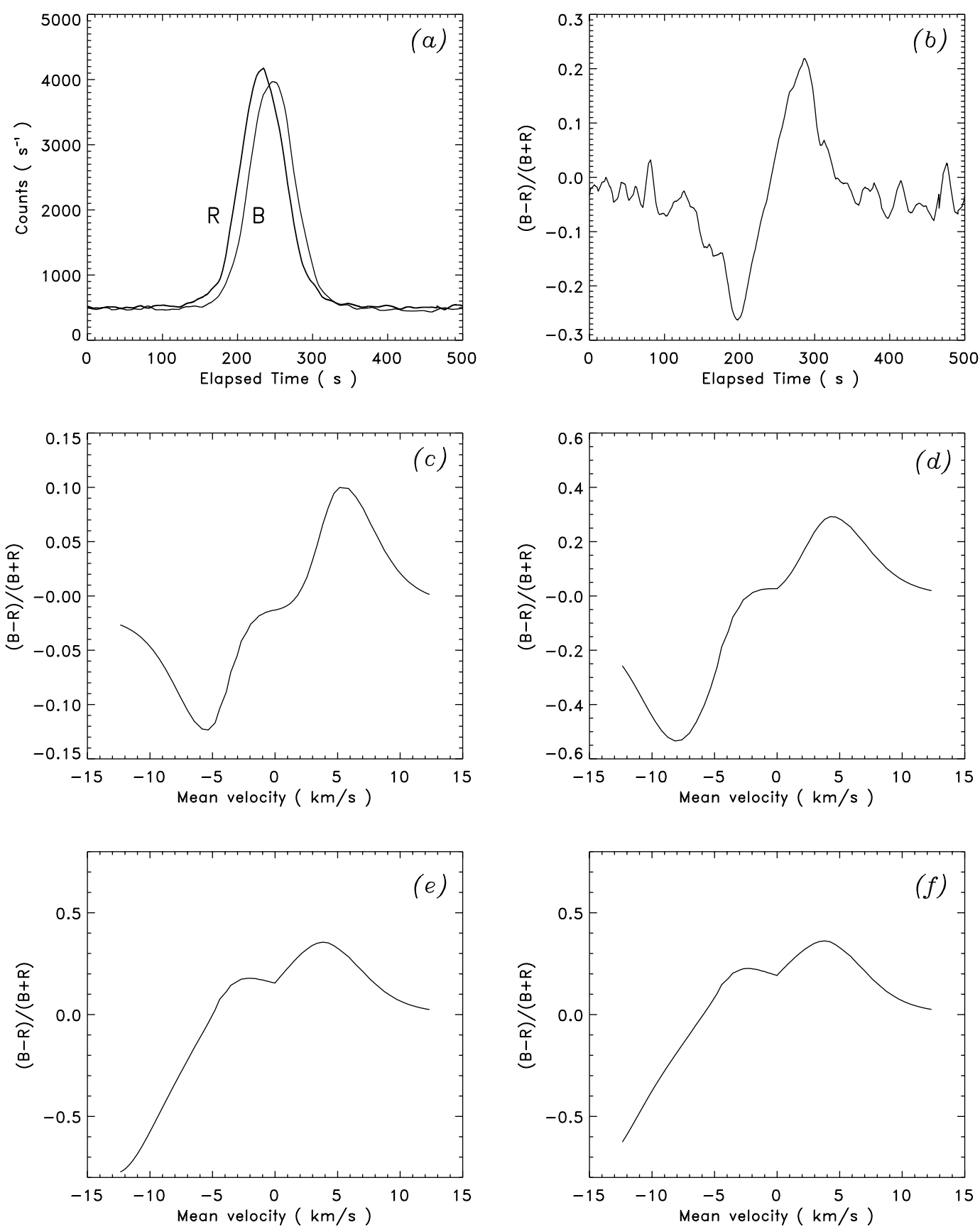

Fig. 4. The passage of Jupiter across the field of view is clearly visible in both $B$ and $R \mathbf{a}$ ); it also produces a characteristic $\mathcal{D}$ curve b). This curve is nearly what is theoretically obtained when Jupiter and the Sun are in opposition as seen from the Earth c). The time in the abscissa is now replaced by the average velocity from the visible portion of the planet. d), e), f) are similar curves for different heliocentric relative longitudes $\left.\left.\left(20^{\circ} \mathbf{d}\right), 50^{\circ} \mathbf{e}\right), 80^{\circ} \mathbf{f}\right)$ ). The ratio between the observed peak amplitudes and the theoretical ones is nearly 2, i.e. the expected factor for observations in reflected sunlight (see also the caption of Fig. 2). This method yields $a_{1} \simeq 10^{-5} \mathrm{~m}^{-1} \mathrm{~s}$.

attention has been given to the optical path so that the risk of vignetting can be excluded.

Figure 4 shows recorded data during the passage of Jupiter across the FOV $(a, b)$ compared with the theoretical variation of $\mathcal{D}$ for four different values of the Earth-Jupiter heliocentric relative longitude (c,d,e,f). The shapes of these curves depend only on the heliocentric relative longitude, whereas their amplitudes depend only on the planet rotation.
The curve computed for the opposition time (Fig. 4c) is in excellent agreement with the observed data (Fig. 4b). Its general structure is evident: it is antisymmetric and increases from negative to positive peak values. This behavior, as the Jovian disk enters the field of view, is the result of two combined effects: a progressive luminosity increase and a simultaneous decrease of the Doppler signal due to the cancellation of the rotation velocity. 
As for numerical details, the ratio between the two peak amplitudes is $\sim 1.25$ for the theoretical estimate and $\sim 1.18$ for the data, in agreement within $6 \%$. Moreover, by comparing the amplitudes of the positive peaks in the data and in the theoretical computation, the values $\sim 0.26$ and $\sim 0.125$ are found. Their ratio, $\sim 2$, is exactly what expected for observations of reflected light.

At the time of our observations $v \simeq-3 \mathrm{~km} \mathrm{~s}^{-1}$ and the curves of Fig. 4 give $a_{1} \simeq 10^{-5} \mathrm{~m}^{-1} \mathrm{~s}$ and $a_{2} \simeq-0.013$ are found. The value of $a_{1}$ is in agreement with Sect. 3.2 while the constant $a_{2}$, it is not important for the study of the frequency spectrum.

The relation between $\mathcal{D}$ and $v$ becomes non-linear beyond about $1 \mathrm{~km} \mathrm{~s}^{-1}$.

\subsection{Other calibration methods}

At least three other calibration methods could be envisaged, but they were not used in this experiment.

First, the data could be calibrated using Doppler images of Jupiter and the known rotation velocity: but such images are not yet available.

Second, the Jovian time series could be combined with other time series from one or more stellar objects whose line profile and velocities are known: by comparing the difference between the signals with the difference between the velocities it is clearly possible to obtain an estimate of the calibration coefficients.

Finally, it is possible to change the wavelength separation of the two MOF transmission bands $R$ and $B$ (by changing the instrument parameters like the temperature and the magnetic field) and to study the consequent variation of the transmitted signal: the application of this method, however, needs a signal-to-noise ratio much higher than usually achieved so far with our small telescope aperture.

In summary, we can conclude that $a_{1} \simeq 10^{-5} \mathrm{~m}^{-1} \mathrm{~s}$ and $b_{1} \simeq 10^{-4} \mathrm{~m}^{-1} \mathrm{~s}$ are the figure numbers to be used to calibrate $\mathcal{D}$ and $\mathcal{S}$ respectively: the determination of the two offsets $a_{2}$ and $b_{2}$ is uncertain, but they can be completely neglected in the Fourier analysis.

\section{Analysis}

\subsection{Structure of the power spectra}

In order to obtain the power spectra of $\mathcal{D}$ and $\mathcal{S}$, the data of 11, 12 and 13 July were joined together, by filling the gaps with zero-values. The corresponding resolution becomes $3.81 \mu \mathrm{Hz}$, but the spectrum is heavily contaminated by the window function, in which missing-data dominate.

Two groups of features can be recognized in the power spectrum of $\mathcal{S}$, shown in Fig. 5a. One is centered around $0.6 \mathrm{mHz}$, the other around $1.6 \mathrm{mHz}$. This spectrum is quite similar to those shown in Fig. 2a by Schmider et al. (1991) and Fig. 10 by Mosser et al. (1993). Indeed the structure around $0.6 \mathrm{mHz}$ is essentially the same and seems probably due to albedo variations on the Jupiter's surface.
(Lederer et al. (1995) suggested that, in this case, the power should be peaked at about $700 \mu \mathrm{Hz}$ ). The structure around $1.6 \mathrm{mHz}$ is present in Schmider et al. (1991) and Mosser et al. (1993) but in our data it is largely reduced in extent: probably it is due to instrumental effects, as we describe in Sects. 4.2-4.3 .

In the spectrum of $\mathcal{D}$ no peaks are detected above a 1- $\sigma$ noise level corresponding to $\sim 1.5 \mathrm{~m}^{2} \mathrm{~s}^{-2} \mathrm{~Hz}^{-1}$.

Echelle diagrams have been computed for both $\mathcal{D}$ and $\mathcal{S}$ spectra, in order to identify the frequencies of the peaks and to clarify their origin: no clear and significant vertical alignments have been found, except for the frequency of the Earth rotation, $11.6 \mu \mathrm{Hz}$, corresponding to about 3 bins in the spectrum of $\mathcal{S}$. However, when adopting some signal recovery analysis from noise, as for example suitable frequency binning to enhance the power in regions where a known pattern of crowded peaks is expected, a small bump in the $3.3 \mathrm{mHz}$ region is discovered. We are waiting for a confirmation from the next observing campaign. If real, this would be the signature of the solar $p$-modes seen in the reflected light from Jupiter, with decisive importance about the reliability and calibration of the observations.

\subsection{The different sensitivity of $\mathcal{D}$ and $\mathcal{S}$ techniques}

A crucial point when searching for Jovian oscillations with the magneto-optical technique is the following discussion of the sensitivity of the different algorithms. With the term "sensitivity" we mean here the deviation of the signals $(\mathcal{D}, \mathcal{S})$ from their "unperturbed" values per unit velocity

$S_{\mathcal{D}}=\left(\mathcal{D}_{\mathrm{p}}-\mathcal{D}_{\mathrm{u}}\right) / \delta v$

$S_{\mathcal{S}}=\left(\mathcal{S}_{\mathrm{p}}-\mathcal{S}_{\mathrm{u}}\right) / \delta v$

where $\delta v$ is the variation of the velocity field on the Jupiter's surface from its "unperturbed" configuration

$\delta v=v_{\mathrm{p}}(\theta, \phi)-v_{\mathrm{u}}(\theta, \phi)$

and $\theta, \phi$ are the zenographic latitude and longitude.

The sensitivities $S_{\mathcal{D}}$ and $S_{\mathcal{S}}$ are not only different one another but also involve the epoch of observation $t_{\text {obs }}$ and the degrees $(\ell, m)$ of the oscillation mode, i.e.

$S_{\mathcal{D}}=f_{\mathcal{D}}{ }^{(\ell, m)}\left(t_{\mathrm{obs}}\right)$

$S_{\mathcal{S}}=f_{\mathcal{S}}{ }^{(\ell, m)}\left(t_{\mathrm{obs}}\right)$.

These functions are discussed in detail in another paper (Dolci \& Cacciani 2001, in preparation). Here we outline only the general results of this analysis. At the opposition, $\mathcal{S}$ displays maximum sensitivity to albedo variations and is insensitive to oscillation modes, while $\mathcal{D}$ is sensitive to oscillations and insensitive to albedo variations. At the quadrature, the situation is reversed.

When applied to our experiment, the above results lead to the following conclusions: since nothing is visible in $\mathcal{D}$ above the noise we can conclude that no oscillation power is present in our data with an amplitude above 

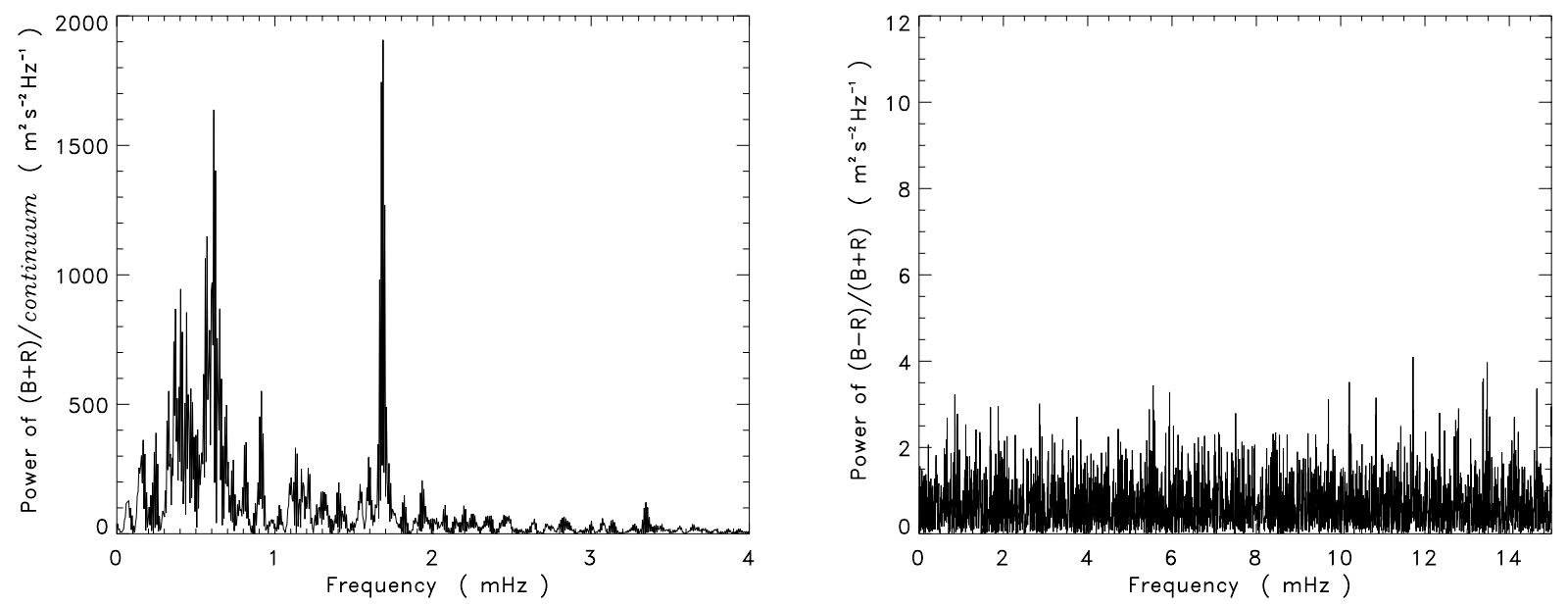

Fig. 5. Power spectra of the calibrated $\mathcal{S}$ (left) and $\mathcal{D}$ (right) signals. In the spectrum of $\mathcal{S}$ two main groups of frequency can be easily identified: the first one is located around $0.6 \mathrm{mHz}$ and is probably due to albedo variations on Jupiter's surface; the second one ranges between 1.5 and $1.8 \mathrm{mHz}$ and should be related to instrumental effects. In the spectrum of $\mathcal{D}$ nothing seems to be detected above a $1-\sigma$ noise level of $\sim 1.5 \mathrm{~m}^{2} \mathrm{~s}^{-2} \mathrm{~Hz}^{-1}$. This led us to the conclusion that any possible oscillation mode on Jupiter should have an amplitude smaller than this limit. See the text for the explanation.

$\sim 1.5 \mathrm{~m}^{2} \mathrm{~s}^{-2} \mathrm{~Hz}^{-1}$. Taking into account the spreading of power due to the window function, we set an upper limit for the oscillation velocities corresponding to $1.2 \mathrm{~m} \mathrm{~s}^{-1}$ in the range between 0.5 and $0.7 \mathrm{mHz}$, where albedo seems to dominate but also real oscillations are theoretically expected.

Regarding the $\mathcal{S}$ spectrum, its peaks cannot be produced by $p$-modes: in particular, the ones around $0.6 \mathrm{mHz}$ are probably due to albedo variations, also because they are not found in the spectrum of $\mathcal{D}$ as expected for the albedo. The peaks around $1.6 \mathrm{mHz}$ are possibly due to instrumental effects as discussed in the next section.

We need to comment here the recent claim of Mosser et al. (2000) that puts a lower limit at $60 \mathrm{~cm} \mathrm{~s}^{-1}$ bet ween 1 and $3 \mathrm{mHz}$ in comparison with our quotation of $1.2 \mathrm{~m} \mathrm{~s}^{-1}$ between 0.5 and $0.7 \mathrm{mHz}$.

First of all, we notice that in Mosser et al. (2000) the performances of the instrument and the different sources of error are discussed in deep detail. Particularly there is considerable effort to optimize the stability. However, the extreme complexity of the instrument and the many parameters to be controlled (some of them produce large noise for small variations, as the guiding, the turbulence and the mechanical instability) leave the reader a little concerned about the full reliability of the calibration achievement.

Second, in our technology we have a much simpler and compact instrument with only one parameter to be controlled, the temperature, that requires only few tenths of ${ }^{\circ} \mathrm{C}$. In addition, we have been using a smaller telescope (72 $\mathrm{cm}$ of Teramo Observatory in comparison with $3.6 \mathrm{~m}$ of the CFH at Mauna Kea). Considering also that the length of the observing run (our 17 hours against 32.8) and the average sky transparency are in favor of Mosser for at least a factor three, the quoted limits (our $1.2 \mathrm{~m} \mathrm{~s}^{-1}$ and Mosser's $0.6 \mathrm{~m} \mathrm{~s}^{-1}$ ) are equivalent or slightly in favor of us as far as the random error level determined by the telescope aperture is concerned (photon statistics), but the present technique should be less prone to systematic errors not easily accounted for.

The two above circumstances make us confident to be able to establish the aimed network with $1 \mathrm{~m}$-class telescopes more easily in the future.

\subsection{Other sources of spurious signal}

Although special care was taken to control the experiment parameters, some possible sources of spurious signal could still have been working and should be examined.

A source of noise could be possible image displacements within the field of view due to tracking errors. In this case the light beam crosses different portions of the metallic vapors inside the MOF, having slightly different conditions of density, temperature and magnetic field. Therefore the transmission profiles of $B$ and $R$ change randomly and the signal fluctuations could have produced the peaks in $\mathcal{S}$. However, this phenomenon should affect the $\mathcal{D}$ spectrum as well, in contrast with what observed.

Temperature fluctuations in the various stages of the acquisition system produce the most dangerous spurious signals. It is well known that temperature changes of the interference prefilter could be responsible for variations in its transmission profile; in the same way, temperature fluctuations of the MOF cells can seriously modify the $B$ and $R$ profiles. This is the reason why in our case the temperature was controlled within $1{ }^{\circ} \mathrm{C}$ in order to keep these sources of noise negligibly small.

However, the most important effect is probably related to temperature fluctuations of the detectors, because of induced changes in their responsivities. As a matter of fact, a measurement of the dark current of the photomultiplier shows characteristic frequencies just in the range around 
Table 1. Expected noise levels in the power spectra of $\mathcal{D}$ and $\mathcal{S}\left(\mathrm{m}^{2} \mathrm{~s}^{-2} \mathrm{~Hz}^{-1}\right)$.

\begin{tabular}{||l|c|c||}
\hline \hline Source & Dnoise & Snoise \\
\hline Photon statistics & $\simeq 1.51$ & $\simeq 3$ \\
\hline Guiding errors & $\simeq 10^{-5}$ & $\simeq 10^{-5}$ \\
\hline $\begin{array}{l}\text { Prefilter temperature } \\
\text { fluctuations }\end{array}$ & $\simeq 10^{-4}$ & $\simeq 10^{-4}$ \\
\hline $\begin{array}{l}\text { MOF cells temperature } \\
\text { fluctuations }\end{array}$ & $\simeq 10^{-2}$ & $\simeq 10^{-2}$ \\
\hline $\begin{array}{l}\text { Dark current } \\
\text { fluctuations }\end{array}$ & $\simeq 3 \times 10^{-3}$ & $\simeq 9 \times 10^{-5}$ \\
\hline Readout noise & $<10^{-7}$ & $<10^{-7}$ \\
\hline \hline
\end{tabular}

$1.6 \mathrm{mHz}$ and this could justify the peaks observed in the $\mathcal{S}$ and not in $\mathcal{D}$. This is exactly what expected assuming independent fluctuations in the responsivities of the photomultiplier (which controls $B$ and $R$ ) and the photodiode (which controls the continuum signal): the fluctuations are canceled out by the ratio in $\mathcal{D}$, but not in $\mathcal{S}$. This excludes also any possible correlation between the various stages of the system, in particular fluctuations in the general power supply.

Table 1 summarizes the noise levels in the power spectra, quoted for the above mentioned sources: apart from the large peaks in the $\mathcal{S}$ spectrum, the photon statistics is expected to dominate both spectra, in agreement with the data.

In conclusion, we can say that the peaks around $1.6 \mathrm{mHz}$ have an instrumental origin. Aware of this, our group is preparing a more refined experimental set-up (with only one detector) and planning longer measurements.

\section{Conclusions}

The observation and the analysis described in this paper represent an important step forward in the understanding the problems involved in the detection of the Jovian oscillations. This will guide us in the preparation and interpretation of future experiments.

Our technique, based on a two-cell magneto-optical filter, allowed us to record three separate signals, $B, R$ and continuum. From them it is possible to compose other convenient combinations as, for example, the two algorythms $\mathcal{D}=(B-R) /(B+R)$ and $\mathcal{S}=(B+R) /$ continuum. They are not affected by the transparency fluctuations of the terrestrial atmosphere.

The two major sources of signal, the oscillations and the albedo fluctuations, have complementary properties in the sensitivity of $\mathcal{D}$ and $\mathcal{S}$. This allows us to analyze almost the full range of possible combinations of albedo and velocity variations.

From the analysis of their power spectra, we have concluded that no clear oscillations are present on Jupiter with amplitudes above the $1-\sigma$ level of $\sim 1.2 \mathrm{~m} \mathrm{~s}^{-1}$ in the range between 0.5 and $0.7 \mathrm{mHz}$.

The signal caused by the albedo fluctuations is indeed present, with an equivalent velocity amplitude of some tens of meters per second. These peaks are in the range predicted by Lederer et al. (1995), around $0.6 \mathrm{mHz}$, and are quite similar to those detected by Mosser et al. (1993) and by Schmider et al. (1991).

Signal enhancement analysis in overwhelming noise does show some power also in the $3-4 \mathrm{mHz}$ region, but this has to be confirmed.

Both signals, $\mathcal{D}$ and $\mathcal{S}$, are sensitive to variations of the experiment parameters. However, while $\mathcal{D}$ has an "internal" normalization factor $(B-R$ is normalized to $B+R), \mathcal{S}$ is normalized to the "external" continuum. For this reason, $\mathcal{S}$ is affected by uncorrelated fluctuations of the responsivities of the photomultiplier (used to detect $B$ and $R$ ) and the photodiode (used to detect the continuum signal). This fact is evident in the power spectrum of $\mathcal{S}$ (which exhibits peaks at about $1.6 \mathrm{mHz}$, the same range of fluctuations of the photomultiplier's dark current) but not in the spectrum of $\mathcal{D}$ where any photomultiplier fluctuation is canceled.

Longer observations, performed with a single detector and at different epochs of the year, are necessary to confirm our conclusions.

Acknowledgements. The authors thank Agostino Di Paolantonio, Barbara Cullmann and Francesca Poli for their help in the preparation and performance of the observations.

They thank also the Ministero dell'Università e della Ricerca Scientifica, the Osservatorio Astronomico "V. Cerulli" of Teramo, the Agenzia Spaziale Italiana (ASI) and the Programma Nazionale di Ricerche in Antartide (PNRA) for financial support.

\section{References}

Bercovici, D., \& Schubert, G. 1987, Icarus, 69, 557

Brown, T. M., \& Gilliland, R. L. 1994, ARA\&A, 32, 37

Cacciani, A., \& Fofi, M. 1978, Sol. Phys., 59, 179

Cacciani, A., Ricci, D., Rosati, P., et al. 1988, Acquisition and reduction procedures for MOF Doppler-Magnetograms, in Seismology of the Sun and Sun-like Stars, ESA-286, 185

Cacciani, A., Rosati, P., Ricci, D., et al. 1991, JPL Internal Report D11900

Cacciani, A., Moretti, P. F., Dolci, M., et al. 1995, Geophys. Res. Lett., 22, 2437

Deming, D., Mumma, M. J., Espenak, F., et al. 1989, ApJ, 343, 456

Deming, D., Reuter, D., Jennings, D., et al. 1997, Icarus, 126, 301

Dolci, M., \& Cacciani, A. 2001, in preparation

Gelly, B., Grec, G., \& Fossat, E. 1986, A\&A, 164, 383

Harrington, J., Dowling, T. E., \& Baron, R. L. 1996a, Icarus, 124,22

Harrington, J., Dowling, T. E., \& Baron, R. L. 1996b, Icarus, 124,32

Innis, J. L., Isaak, G. R., Speake, C. C., et al. 1991, A\&A, 249, 643 
Innis, J. L., Isaak, G. R., Speake, C. C., et al. 1994, A\&A, 271, 573

Lederer, S. M., Marley, M. S., Mosser, B., et al. 1995, Icarus, 114,269

Lee, U. 1993, ApJ, 405, 359

Lee, U., Strohmayer, T. E., \& Van Horn, H. M. 1992, ApJ, 400, 362

Magalhães, J. A., Weir, A. L., Conrath, B. J., et al. 1989, Nature, 337, 444

Magalhães, J. A., Weir, A. L., Conrath, B. J., et al. 1991, Icarus, 88, 39

Moretti, P. F., Cacciani, A., Dolci, M., \& Giuliani, C. 1999, BAAS, 31, 1156

Mosser, B. 1990, Icarus, 87, 198

Mosser, B. 1995, A\&A, 293, 586
Mosser, B., Schmider, F. X., Delache, Ph., \& Gautier, D. 1991, A\&A, 251, 356

Mosser, B., Mékarnia, D., Maillard, J. P., et al. 1993, A\&A, 267, 604

Mosser, B., Gudkova, T., \& Guillot, T. 1994, A\&A, 291, 1019

Mosser, B., Maillard, J. P., \& Mékarnia, D. 2000, Icarus, 144, 104

Pirraglia, J. A. 1992, Icarus, 96, 161

Rhodes, E. J., Jr., Cacciani, A., Korzennik, S., et al. 1990, ApJ, 351,687

Schmider, F. X., Mosser, B., \& Fossat, E. 1991, A\&A, 248, 281

Tomczyk, S., Streander, K., Card, G., et al. 1995, Sol. Phys., 159,1

Vorontsov, S. V., Zharkov, V. N., \& Lubimov, V. M. 1976, Icarus, 27, 109 